\title{
Étude de la circulation de la Cena Cypriani durant le Moyen-Âge
}

(avec édition de textes)

octorat en histoire et civilisation, sous la direction de Marie Anne Polo

de Beaulieu, École des hautes études en sciences sociales (EHESS), soutenu le 24 octobre 2011.

\section{Andrea Livini}

\section{(2) OpenEdition}

\section{Journals}

Édition électronique

URL : http://journals.openedition.org/assr/24591

DOI : $10.4000 /$ assr. 24591

ISSN : $1777-5825$

Éditeur

Éditions de l'EHESS

Édition imprimée

Date de publication : 30 décembre 2012

Pagination : 309-358

ISSN : 0335-5985

Référence électronique

Andrea Livini, «Étude de la circulation de la Cena Cypriani durant le Moyen-Âge », Archives de sciences sociales des religions [En ligne], 160 | octobre-décembre 2012, mis en ligne le 14 mars 2013, consulté le 20 avril 2019. URL : http://journals.openedition.org/assr/24591

Ce document a été généré automatiquement le 20 avril 2019

(c) Archives de sciences sociales des religions 


\section{Étude de la circulation de la Cena Cypriani durant le Moyen-Âge}

(avec édition de textes) octorat en histoire et civilisation, sous la direction de Marie Anne Polo de Beaulieu, École des hautes études en sciences sociales (EHESS), soutenu le 24 octobre 2011.

\section{Andrea Livini}

1 Le sujet principal de notre thèse doctorale est l'étude de la Cena Cypriani, texte chrétien en prose, écrit au $\mathrm{v}^{\mathrm{e}}$ siècle, probablement en France, qui, bien que nous ignorions l'identité de son auteur, fut attribué à Cyprien de Carthage (200-258 après J.-C.) au cours du haut Moyen Âge.

2 La Cena Cypriani est le court récit d'un singulier banquet biblique, organisé par le roi Joël (l'image de Dieu) auquel sont conviés tous les principaux personnages de la Bible, d'Adam à Jésus-Christ. Alors que les invités préparent le banquet, se servent les uns les autres et s'amusent jusqu'à l'ivresse, soudainement, en raison du vol mystérieux des biens précieux du roi Joël, ils sont tous soupçonnés et soumis à la torture. Une fois découvert l'auteur du vol, les autres convives sont remis en liberté et peuvent rentrer chez eux.

3 L'on peut remarquer qu'au ton ouvertement satirique du texte, s'ajoute une technique narrative unique: le texte est constitué d'épisodes bibliques qui, par un mécanisme associatif, sont liés à des objets réels (comme la nourriture). Ce mécanisme devait sans doute permettre de comprendre les nombreuses et indirectes citations bibliques contenues dans le texte, ce qui conférait à la Cena Cypriani une certaine fonction didactique.

$4 \quad$ L'élaboration de cette thèse a supposé un double travail : étudier la tradition originelle du texte - et ses principales réélaborations médiévales - et étudier l'importance de la Cena Cypriani au Moyen Âge.

5 Nous avons divisé notre travail en deux parties et également proposé, dans un appendice, l'édition de textes, objet d'étude de notre travail. 
6 Pour établir le texte de la Cena Cypriani, nous avons tout d'abord proposé une étude des codices ayant transmis le texte (qui sont au nombre de soixante), et qui ont été écrits notamment en France entre le $\mathrm{IX}^{\mathrm{e}}$ siècle et le $\mathrm{XII}^{\mathrm{e}}$ siècle.

7 À cette étude l'on a fait suivre un travail consacré aux sources du texte, qui permet de mieux comprendre la fonction de la Cena Cypriani au Moyen Âge.

8 Nous avons donc tâché d'étudier, dans un premier temps, la création littéraire du texte grâce à la reconstitution de la "bibliothèque » de l'auteur anonyme qui maîtrisait parfaitement la langue grecque et connaissait un grand nombre d'auteurs latins antiques (notamment Pline et Martial) et tardifs (Ammien Marcellin et Végèce) puis, dans un second temps, le milieu social et culturel dans lequel cet auteur aurait pu écrire la Cena Cypriani et surtout la fonction de cette dernière. Nous avons pu conjecturer que la Cena Cypriani était un texte de divertissement chrétien qui servait, lors de lectures publiques ou privées, à amuser l'auditeur ou le lecteur. En effet, dès le $\mathrm{V}^{\mathrm{e}}$ siècle la fonction principale du texte est celle qui lui sera encore attribuée au Moyen Âge: il s'agit d'amuser, tout en enseignant la Bible aux auditeurs et aux lecteurs par le biais d'indirectes et nombreuses références bibliques qui constituent des images faciles à mémoriser et performer. La Cena Cypriani présente également de complexes allégories qui font penser que ce texte chrétien pouvait avoir de précises significations religieuses et exégétiques. Elle est un unicum dans la littérature latine tardive, même si l'on peut retrouver dans d'autres textes analogues $\mathrm{du}_{\mathrm{IV}}^{\mathrm{e}}$ siècle, comme le Iudicium coci et pistoris et le Testamentum porcelli, textes liés à l'inversion comique et religieuse de la fête des Saturnalia, ce type de didactique comique.

9 Dans la seconde partie de ce travail, nous avons proposé une raison à la fortune de la diffusion de la Cena Cypriani au début du haut Moyen Âge, notamment en France : elle offrait une nouvelle façon de comprendre et d'apprendre la Bible, tant aux élèves des écoles qu'aux personnes illettrées, puisque elle était à la base de la mise en scène de processions formées par des personnes déguisées en personnages bibliques. Nous avons, en outre, dans cette même partie, démontré que la Cena Cypriani originale est l'un des archétypes des mystères médiévaux et qu'elle est également un texte d'importance fondamentale pour comprendre la performance religieuse médiévale.

10 La circulation du texte et sa fortune sont marquées - notamment au haut Moyen-Âge - par les deux réélaborations qui en ont été faites au IX ${ }^{\mathrm{e}}$ siècle : la Cena Nuptialis écrite en 856 par l'ancien abbé de Fulda, Raban Maure (780-856), et la Cena Iohannis de Jean Inmonides (IX ${ }^{\mathrm{e}}$ siècle), diacre romain faisant partie de la cour papale de Jean VIII (872-882) qui transforma le texte original en un poème ayant pour finalité principale celle d'être représenté à Rome, pendant la fête de Pâques, par le biais d'une procession religieuse et de rituels d'inversion comiques représentant un mystère sacerdotal. Cette représentation romaine était une imitation de l'usage didactique et religieux carolingien de la Cena Cypriani. Lors de l'élaboration de notre édition critique de la Cena Iohannis nous avons découvert que c'est Charles le Chauve (823-877), qui se rendit à Rome en 875 pour être couronné empereur, qui fit découvrir la Cena Cypriani aux Romains, en l'utilisant pour réaliser une procession religieuse de type théâtral.

11 La Cena Cypriani était considérée à l'Âge carolingien comme un texte didactique et sa performance liturgique nous ouvre d'intéressantes perspectives pour mieux comprendre la dimension populaire de certaines formes de liturgie théâtrale qui servaient à apprendre la Bible aux masses. Ainsi, la Cena Azelini ( $\mathrm{XI}^{\mathrm{e}}$ siècle) aurait été écrite pour 
réaliser ce type de fonction. Même si elle ne nous est parvenue que de façon incomplète, de nombreux éléments prouvent que cette réélaboration de la Cena Cypriani a été écrite pour être lue publiquement ou pour être chantée, puisqu'elle était accompagnée d'annotations musicales. Par la suite, la Cena Cypriani a encore été réélaborée à deux reprises entre le $\mathrm{XII}^{\mathrm{e}}$ siècle et le $\mathrm{XIII}^{\mathrm{e}}$ siècle, et il existe également un commentaire du texte, écrit par Hervé de Bourg-Dieu, témoignant de la grande importance religieuse de la Cena Cypriani et qui servait également à mieux expliquer les indirectes citations bibliques du texte. En accompagnant la Cena Cypriani, il facilitait probablement aux lecteurs la compréhension et la mémorisation logique du texte original. Cette didactique, qui peut sembler originale, eut une grande importance dans les écoles monastiques à partir de l'Âge carolingien : en effet, la Cena Cypriani médiévale est majoritairement transmise par les manuscrits provenant des scriptoria monastiques, notamment bénédictins et cisterciens. L'écriture et la transmission de la Cena Cypriani au Moyen Âge semblent justifiées par la fonction didactique du texte, une didactique de la Bible complexe, mais efficace, qui se faisait également grâce aux réécritures du texte. Parmi celles-ci, il faut compter l'une des dernières réélaborations connues - sans doute la plus complexe - la Cena d'Arras. Cette réélaboration, bien qu'elle ne nous soit parvenue que de façon incomplète et même si l'on peut douter qu'elle ait été représentée publiquement, comme la Cena Iohannis et peut-être la Cena Azelini, est la preuve de l'importance didactique et religieuse de la Cena Cypriani au XIII ${ }^{\mathrm{e}}$ siècle. Cette étude, consacrée à la tradition médiévale de la Cena Cypriani, que nous avons pu réaliser grâce aux nombreux manuscrits qui transmettent la Cena Cypriani et ses réélaborations et réécritures, nous a permis de mettre en lumière une partie méconnue de la didactique de la Bible et de la liturgie médiévale, toutes deux liées au folklore et à la culture populaire.

Pour finir, nous avons proposé dans un appendix une nouvelle édition critique de la Cena Cypriani et de la Cena Iohannis, ce dernier texte étant d'une extrême importance pour comprendre le sujet de la performance publique de thèmes religieux. Nous avons également proposé dans cet appendix une exégèse complète des cinq cents références bibliques indirectes du texte original, une liste des sources qui inspirèrent l'écriture de la Cena Cypriani originale et les textes de la Cena Azelini et de la Cena d'Arras.

\section{AUTEUR}

\section{ANDREA LIVINI}

nomepresioneis@hotmail.com 